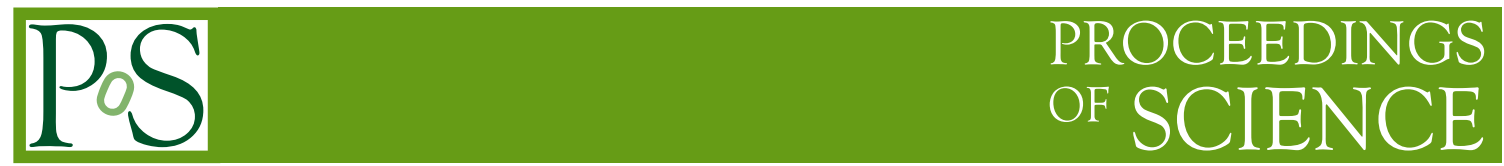

\title{
B-physics studies for HL-LHC ATLAS upgrade
}

\author{
Tomas Jakoubek, on behalf of the ATLAS collaboration \\ Institute of Physics of the Academy of Sciences of the Czech Republic \\ E-mail: tomas.jakoubek@cern.ch
}

\begin{abstract}
Performance studies are made to estimate the ATLAS potential in $B$-physics after upgrade for Run2 and HL-LHC. Real data as well as Monte Carlo simulations are used to study the decay of $B_{s}^{0} \rightarrow J / \psi \phi$ in order to measure the $C P$ violating mixing phase and the width difference between the $B_{s}^{0}$ eigenstates. The increased sensitivity is expected mainly due to the improved decay time resolution obtained with the upgraded IBL and ITk inner tracking detector.
\end{abstract}

The 15th International Conference on Flavor Physics \& CP Violation

5-9 June 2017

Prague, Czech Republic 


\section{Introduction}

The $B_{s}^{0} \rightarrow J / \psi \phi$ decay channel is expected to be sensitive to new physics contributions. In this channel, $C P$ violation occurs due to interference between direct decays and the decays occurring through $B_{s}^{0}-\overline{B_{s}^{0}}$ mixing. The frequency of this mixing is characterized by the mass difference $\Delta M_{S}$ between light $\left(B_{L}\right)$ and heavy $\left(B_{H}\right)$ mass eigenstates. The difference between the mixing and decay amplitudes can be described using a $C P$-violating phase $\phi_{s}$. Assuming no physics beyond the Standard Model (SM) contributions to the $B_{s}^{0}$ mixing and decays, a value of $\phi_{s}=-0.0363_{-0.0015}^{+0.0016} \mathrm{rad}$ is predicted [1]. Other physical quantity involved in $B_{s}^{0}-\overline{B_{s}^{0}}$ mixing is the decay width difference $\Delta \Gamma_{s}=\Gamma_{L}^{s}-\Gamma_{H}^{s}$, where $\Gamma_{L}^{s}$ and $\Gamma_{H}^{s}$ are the decay widths of the different eigenstates. Many new physics models predict larger $\phi_{s}$ values whilst satisfying all existing constraints, including the precisely measured value of $\Delta M_{s}$.

Current experimental results (combined value $\phi_{s}=-0.021 \pm 0.031 \mathrm{rad}[2]$ ) show no significant deviation from the SM prediction, however their uncertainties still leave large room for the new physics (see Figure 1). It is important to note that the systematic as well as the statistical uncertainties will benefit from additional data delivered by the LHC during Run2 and Run3. On the other side, it will be necessary to cope with the very high pile-up provided by the planned High Luminosity LHC (HL-LHC) [3] in Run4 and later.

The potential for future measurements of $C P$ violation in the channel $B_{s}^{0} \rightarrow J / \psi \phi$ on ATLAS relies heavily on the uncertainty of the measured proper decay time. The comparison between Monte Carlo (MC) simulations and the real data is presented here.

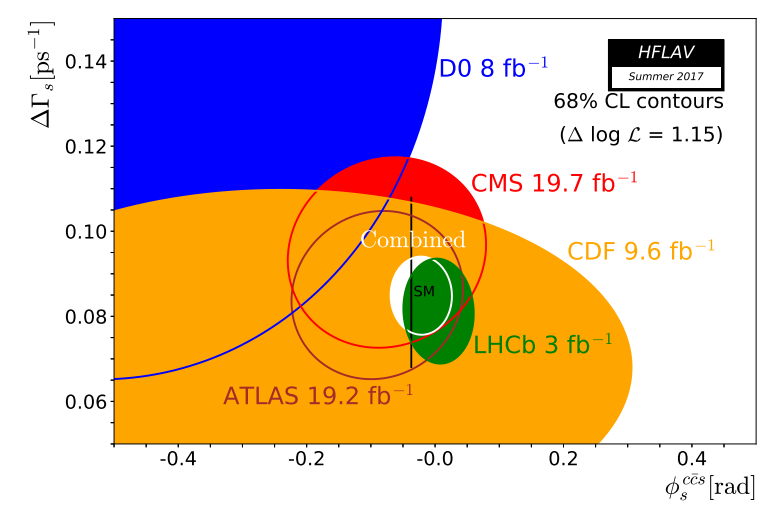

Figure 1: Plot of the $68 \%$ confidence-level contours in the $\phi_{s}-\Delta \Gamma_{s}$ plane with the individual contours of ATLAS, CMS, CDF, D0, and LHC $b$ experiments, their combined contour (white solid line and shaded area), as well as the Standard Model predictions (thin black rectangle) [2].

\section{ATLAS upgrade}

The ATLAS experiment [4] is a multipurpose particle physics detector at the LHC, with a forward-backward symmetrical cylindrical geometry with almost $4 \pi$ coverage. Precise tracking is provided by the Inner Detector (ID) immersed in a $2 \mathrm{~T}$ axial magnetic field. This part is enclosed by the electromagnetic and hadronic calorimeters, followed by the Muon Spectrometer (MS), all located within the magnetic field produced by three large superconducting air-core toroid systems. 
ID consists of a silicon pixel detector, a silicon microstrip detector and a transition radiation tracker. During the LS1 shutdown, a new Insertable B-Layer (IBL) was added to the present ID. It is the fourth layer with a radius $33 \mathrm{~mm}$, inserted between a new beam pipe and the current inner pixel layer (B-layer). IBL will be active until the complete replacement of ID before HL-LHC.

In the next phase the ID will be replaced by a fully silicon-based tracker (ITk) with higher granularity and wider coverage [5]. It is designed to yield at least 13 clusters per charged particle in the barrel part and to provide uniform coverage in the endcap regions. It will allow to provide better tracking and momenta measurement and good vertexing in increased pile-up conditions. ITk layout is shown on the Figure 2.

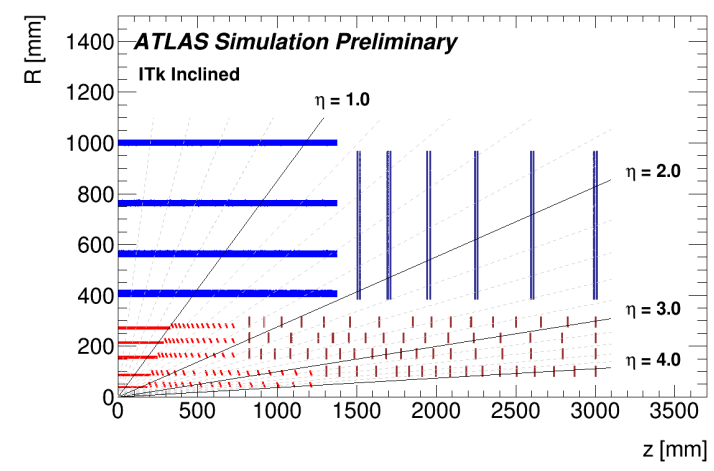

Figure 2: Layout of the ITk detector, shown in the R-z plane. The Pixel tracker is in red, while the Strip tracker is blue [5].

\section{Used data and candidate selection}

For the study presented here, $\sqrt{s}=13 \mathrm{TeV}$ data from proton-proton collisions collected with the ATLAS detector during Run2 are used $\left(3.2 \mathrm{fb}^{-1}\right.$ from 2015 and $18.8 \mathrm{fb}^{-1}$ from 2016). Also results from Run1 (14.3 $\mathrm{fb}^{-1}$ of $\sqrt{s}=8 \mathrm{TeV}$ ATLAS data from 2012) are used for comparison.

MC events are generated for $B_{s}^{0} \rightarrow J / \psi\left(\mu^{+} \mu^{-}\right) \phi\left(K^{+} K^{-}\right)$and $B_{s}^{0} \rightarrow \mu^{+} \mu^{-}$decay channels in three run conditions: Run $1(\sqrt{s}=8 \mathrm{TeV}$ with the mean of the number of interactions per bunchcrossing $\langle\mu\rangle \sim 20$ ), Run2 (IBL, $\sqrt{s}=13 \mathrm{TeV},\langle\mu\rangle \sim 20$ ), and HL-LHC (ITk, $\sqrt{s}=14 \mathrm{TeV}$, $\langle\mu\rangle \sim 200)$.

In the $B_{s}^{0} \rightarrow J / \psi \phi$ decay channel, events are accepted for further processing if they contain at least one reconstructed primary vertex (PV), formed from at least four ID tracks, and at least one pair of oppositely charged muons reconstructed using ID and MS (so-called "combined" muons). Pairs of oppositely charged muon tracks are refitted to a common vertex and accepted if $\chi^{2} /$ n.d.f. $<10$. To account for detector-dependent mass resolution, different $J / \psi$ mass cuts are applied according to $|\eta(\mu)|$. Decays $\phi \rightarrow K^{+} K^{-}$are reconstructed from all pairs of oppositely charged particles with $p_{\mathrm{T}}>1 \mathrm{GeV}$ and $|\eta|<2.5$ that are not identified as muons. Candidates for $B_{s}^{0}$ are selected by fitting the four tracks to a common vertex with $J / \psi$ mass constraint [9]. Candidate is accepted if the vertex fit has $\chi^{2} /$ n.d.f. $<3$ and $\left|m\left(K^{+} K^{-}\right)-M(\phi)\right|<11 \mathrm{MeV}$, where the mass $M(\phi)$ is taken from [9]. If there is more than one accepted $B_{s}^{0}$ candidate in the event, the 
one with the lowest $\chi^{2} /$ n.d.f. is selected. An additional cut $p_{\mathrm{T}}\left(K^{ \pm}\right)>5.5 \mathrm{GeV}$ is applied in MC events.

For the $B_{s}^{0} \rightarrow \mu^{+} \mu^{-}$MC events, $B_{s}^{0}$ candidates are reconstructed from oppositely charged combined muons pairs, with $p_{\mathrm{T}}\left(\mu^{ \pm}\right)>5.5 \mathrm{GeV}$. A vertex fit is performed and the event is accepted if $\chi^{2} /$ n.d.f. $<6$.

\section{Mass and proper decay time uncertainty}

For each $B_{s}^{0} \rightarrow J / \psi \phi$ candidate the proper decay time $t$ is calculated as

$$
t=\frac{L_{x y} M\left(B_{s}^{0}\right)}{p_{\mathrm{T}}\left(B_{s}^{0}\right)},
$$

where $p_{\mathrm{T}}\left(B_{s}^{0}\right)$ is the reconstructed transverse momentum of the $B_{s}^{0}$ meson and $M\left(B_{s}^{0}\right)$ is the mass of the $B_{s}^{0}$ meson, taken from [9]. The transverse decay length $L_{x y}$ is the displacement in the transverse plane of the $B_{s}^{0}$ meson decay vertex with respect to the PV, projected onto the direction of the $B_{s}^{0}$ transverse momentum. The proper decay time uncertainty $\sigma_{t}$ is calculated per-candidate by propagating the uncertainties in track and PV parameters as well as the uncertainties from the $B_{s}^{0}$ candidate decay vertex fit.

Comparison of the three different ID layouts (Run1, Run2 with IBL, HL-LHC with ITk) for the $B_{s}^{0}$ proper decay time resolution $\sigma_{t}$ and the $B_{s}^{0}$ mass resolution $\sigma_{\text {mass }}$ in the simulated MC data are shown on the Figure 3.
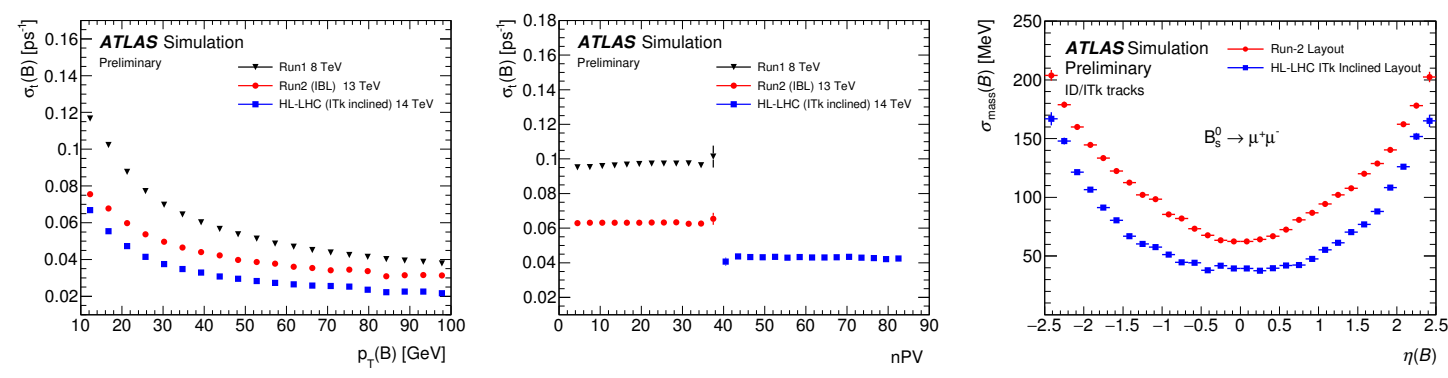

Figure 3: Left and middle plots show the $B_{s}^{0}$ proper decay time resolution $\sigma_{t}$ as functions of the $B_{s}^{0}$ transverse momentum (left) and the number of reconstructed PV (middle). The vertical axis gives the average value of $\sigma_{t}$ for the $B_{s}^{0}$ candidates in the given bin [7]. The right plot shows the $B_{s}^{0}$ mass resolution $\sigma_{\text {mass }}$ as a function of the $B_{s}^{0}$ meson pseudo-rapidity in the decay channel $B_{s}^{0} \rightarrow \mu^{+} \mu^{-}$. The resolution is defined as RMS of the histograms of difference between the reconstructed and generated invariant masses in the given bin [8].

To study the proper decay time uncertainty in the real data, a sideband-subtraction method is used. A fraction between signal and background events is extracted from a fit to the $B_{s}^{0}$ mass distribution. The background component of the $\sigma_{t}$ distribution is fitted in the $B_{s}^{0}$ mass sidebands, (5.150-5.317) $\mathrm{GeV}$ or (5.417-5.650) GeV, using an unbinned maximum likelihood fit (UMLF). A signal component is then obtained from the UMLF to all events, where the function describing the $\sigma_{t}$ background and the signal fraction are fixed. The $B_{s}^{0}$ proper decay time uncertainty distribution extracted from the $B_{s}^{0} \rightarrow J / \psi \phi$ decay candidates in 2012, 2015, and 2016 data are shown on the Figure 4. Improvement in the signal part of the distribution is clearly visible there. 

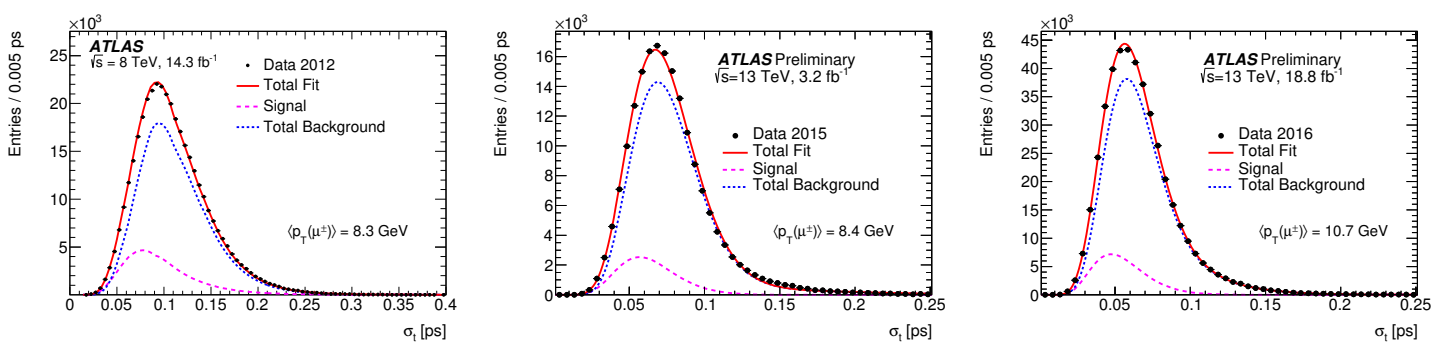

Figure 4: The $B_{s}^{0}$ proper decay time uncertainty distribution extracted from the $B_{s}^{0} \rightarrow J / \psi \phi$ decay candidates in Run $18 \mathrm{TeV}$ data collected in 2012 (left) and Run2 $13 \mathrm{TeV}$ data collected in 2015 (middle) and 2016 (right) respectively. Data are shown as points and results of fit to signal, background, and the total fit are shown as lines. Left plot is taken from [6], middle and right plots from [7].

\section{Summary}

Performance studies are made to estimate the ATLAS potential in $B$-physics after upgrade for Run2 and HL-LHC.

MC simulations are used to estimate the $B_{s}^{0}$ mass resolution in the $B_{s}^{0} \rightarrow \mu^{+} \mu^{-}$channel. As shown on Figure 3, it strongly depends on the pseudo-rapidity of the reconstructed $B_{s}^{0}$. An improvement by a factor of about 1.65 in barrel and 1.50 in endcap region is expected after the upgrade from current ID with IBL to completely new ITk.

Real data as well as Monte Carlo simulations are used to study the decay of $B_{s}^{0} \rightarrow J / \psi \phi$, which is expected to be sensitive to new physics contributions. Precision of measurement of $C P$-violating parameters rely heavily on the proper decay time uncertainty. It is improved in Run2 with the upgraded IBL and it is expected to get even better with ITk inner tracking detector during HL-LHC operation.

\section{Acknowledgments}

I would like to thank for support from grants of the Ministry of Education, Youth and Sports of the Czech Republic under the project LG15052.

\section{References}

[1] J. Charles et al., Predictions of selected flavour observables within the Standard Model, Phys. Rev. D 84 (2011) 033005.

[2] Heavy Flavour Averaging Group, Averages of b-hadron, c-hadron, and $\tau$-lepton properties as of summer 2016, arXiv:1612.07233 [hep-ex].

[3] HL-LHC High Luminosity Large Hadron Collider: The HL-LHC project, (Accessed June 2017), http://hilumilhc.web.cern.ch/about/hl-lhc-project.

[4] The ATLAS Collaboration, The ATLAS Experiment at the CERN Large Hadron Collider, JINST 3 (2008) S08003.

[5] The ATLAS Collaboration, Expected Performance of the ATLAS Inner Tracker at the High-Luminosity LHC, ATL-PHYS-PUB-2016-025, https://cds . cern. ch/record/2222304. 
[6] The ATLAS Collaboration, Measurement of the CP-violating phase $\phi_{s}$ and the $B_{s}^{0}$ meson decay width difference with $B_{s}^{0} \rightarrow J / \psi \phi$ decays in ATLAS, JHEP 1608 (2016) 147.

[7] The ATLAS Collaboration, $B_{s}^{0}$ proper decay time resolution in the $B_{s}^{0} \rightarrow J / \psi\left(\mu^{+} \mu^{-}\right) \phi\left(K^{+} K^{-}\right)$ decay for Run-1, Run-2 and HL-LHC, (Accessed June 2017), https : / / atlas . web. cern . ch/ Atlas/GROUP S / PHYS ICS / PLOTS / BPHYS-2016-001/index.html.

[8] The ATLAS Collaboration, Expected performance for an upgraded ATLAS detector at High-Luminosity LHC, ATL-PHYS-PUB-2016-026, http://cdsweb.cern.ch/record/2223839.

[9] The Particle Data Group Collaboration, Review of Particle Physics, Chin. Phys. C 40 (2016), 100001. 